\title{
Doble Graduación en Cirugía Oral y Máxilofacial
}

\author{
Dual-Degree Oral and Maxillofacial Surgery
}

\author{
Ricardo Cartes-Velásquez* \& Hernán Ramírez Skinner
}

CARTES-VELÁSQUEZ, R. \& RAMíREZ, S. H. Doble graduación en cirugía oral y máxilofacial. Int. J. Odontostomat., 5(2):191-198, 2011.

RESUMEN: Las patologías orales y máxilofaciales son tratadas por especialidades médicas y quirúrgicas que se superponen, la mayoría de ellas sin la formación de alto nivel en estomatología que posee el cirujano dentista. De estas especialidades, la Cirugía Oral y Máxilofacial es la única que requiere formación médica, dental y quirúrgica, que actualmente es alcanzada a través de 3 rutas principales. Hoy, continua el debate acerca de cual de ellas es la ideal, aunque en América Latina la única vía para convertirse en especialista es la odontológica. Se realizó una revisión crítica de la literatura internacional en revistas de la especialidad y guías de las sociedades internacionales, enfocándose en los aspectos bioéticos, educativos, legales, sociales y de competencias clínicas. La doble-graduación es ventajosa para la práctica de la especialidad en los siguientes aspectos: campos de acción clínico (cirugía oncológica y reconstructiva), acceso a subespecialidades y sociedades científico-profesionales, generación de conocimientos/evidencia, prerrogativas quirúrgicas, sustento legal y reconocimiento social. Desde una perspectiva bioética, el especialista de un solo grado que trata la patología máxilofacial podría estar excediendo sus competencias debido a la falta de formación médica u odontológica. El especialista doble graduado parece ser quien, desde una visión integral del paciente, tiene los conocimientos y habilidades para satisfacer completamente el derecho a la salud de la comunidad y contribuir al desarrollo de la especialidad. Lamentablemente, en América Latina no existen programas de formación de doble grado y ninguno de los chilenos cumple a cabalidad con los requisitos planteados en las guías internacionales. Esta revisión expone la necesidad de desarrollar un programa de formación de doble grado en Chile para alcanzar una aproximación integral del paciente con patología máxilofacial, en coherencia con la realidad social, cultural, económica y educacional del país.

PALABRAS CLAVE: cirugía, máxilofacial, doble graduación, revisión, Chile.

\section{INTRODUCCIÓN}

Desde tiempos inmemoriales las prácticas tendientes a curar la enfermedad y recuperar la salud han estado asignadas a actores específicos dentro de las diversas sociedades, primero sustentadas en explicaciones divinas y/o sobrenaturales hasta evolucionar a aquellas basadas en el método científico (MorenoAltamirano, 2007; Cruz-Coke, 2007), recayendo sobre estos actores una gran responsabilidad y reconocimiento social.

En los primeros grupos humanos este rol generalmente lo jugaba un solo individuo, pero posteriormente, dado el desarrollo técnico y/o científico de estas prácticas, comienzan a aparecer tareas específicas que son realizadas por distintas personas. Esta división de roles que puede parecer bastante obvia no está influenciada por factores puramente técnicos, si no también sociales y políticos. Así vemos que la concepción actual de la medicina y la odontología no es un status quo que se haya mantenido desde las antiguas grandes civilizaciones, por el contrario ha sido el resultado de un dinámico proceso histórico que es diferente en cada cultura o región (Schapira, 2003).

Actualmente vemos que la medicina que se enseña es un agregado de la medicina propiamente tal y la cirugía, sumando de una forma singular a la farmacología, pero excluyendo a la odontología. Dicha exclusión también obedece a un proceso histórico que presenta variaciones importantes según el país, con 2 modelos imperantes: la odontología y la estomatología, la primera como una carrera totalmente se-

\footnotetext{
* Programa de Doctorado en Ciencias Médicas, Facultad de Medicina, Universidad de la Frontera, Temuco, Chile.

" Departamento de Cirugía Oncológica y Máxilofacial, Facultad de Medicina, Pontificia Universidad Católica de Chile, Santiago, Chile.
} 
parada de la medicina y la segunda como una especialidad de ella (Scott, 2003), aunque encontramos casos donde estos términos se mezclan y hasta contradicen (Legrá Matos et al., 2006).

Para complicar aún más estas divisiones en los campos de acción, vemos que la odontología también ha desarrollado clínicamente un enfoque médico y otro quirúrgico para el tratamiento de las patologías que a ella le compete, para variar, esas competencias van desde una visión dental (generalmente asociada a la odontología) hasta una máxilofacial (asociada principalmente a la estomatología), según cada país. Pero en general vemos que el enfoque predominante de la práctica odontológica ha sido el tratamiento de todas las afecciones del diente y sus estructuras inmediatamente cercanas, lo que en el ámbito quirúrgico primordial se ha traducido y limitado principalmente a la "exodoncia" y procedimientos asociados.

Lo anterior se vio fuertemente transformado con las consecuencias de la primera guerra mundial, en ella, miles de heridos y secuelados de traumas faciales presentaban un compromiso de los huesos maxilares y dientes que obligó a sumar a cirujano dentistas en el tratamiento conjunto con médicos (Langdon, 2006). Es aquí donde comienza a tomar un cuerpo más definido la especialidad de "Cirugía Oral y Máxilofacial". Pero al igual que con el resto de la práctica médica, las definiciones frente a que actor sería el encargado de desarrollarla presenta variaciones según el lugar. Todo lo anterior determinó que en un principio un número importante de cirujanos orales y máxilofaciales (COMS) tuviesen una calificación médica y odontológica (Mahmood et al., 2002), la doble graduación, pero posteriormente cada país definió caminos distintos para alcanzar la especialidad, por lo que actualmente nos encontramos con 3 vías principales: la médica, la odontológica y la doble graduación (Laskin, 2008).

Dadas estas desigualdades en la formación de COMS, han surgido, desde un comienzo, dudas acerca de cual vía es la mejor para convertirse en especialista y tratar a los enfermos que le competen, pero en especial determinar la importancia y necesidad de contar con la doble graduación para el ejercicio de la especialidad.

El objetivo del presente artículo es hacer una revisión de la literatura actual, últimos 15 años, respecto a la investigación, realidad y posiciones sobre la doble graduación en cirugía oral y máxilofacial alrede- dor del mundo, así como también analizar una presunta necesidad y factibilidad de desarrollar esta vía de formación en nuestro país.

Formación en el mundo. Actualmente en el plano internacional encontramos diferentes regiones dominadas por alguna de las 3 vías de formación mencionadas anteriormente.

Así es como en América, África y Asia Pacífico (agregar también algunos casos de países europeos) la formación es prácticamente en su totalidad de base odontológica (Laskin), la salvedad a lo anterior se ha estado produciendo en los últimos 40 años con la aparición de los programas de doble graduación en Norteamérica (Dodson et al., 2004), con lo que en estos momentos la mitad los programas en Estados Unidos son dual-degree y el resto single-degree (Assael, 2007); en América Latina solo contamos con la segunda opción.

Una segunda área esta conformada por algunos países europeos donde la formación es de base médica, los más destacados son: España, Francia, Austria e Italia (Goudot \& Bonnel, 2006; Pitak-Arnnop et al., 2010). En ellos vemos que el acceso a los programas de formación es solo para médicos y en general incluyen una base inicial de formación quirúrgica general y/o estomatológica. Aquí también incluimos a vías de formación en que la cirugía oral y máxilofacial es una subespecialidad de la estomatología, como era el caso de España hace unos años o Portugal en la actualidad (UEMS, 2007).

La tercera área la componen otros países europeos en que la doble graduación es obligatoria, entre ellos los más importantes son: Reino Unido, Holanda, Suiza y Alemania. En ellos, el acceso implica que los postulantes deban tener el título de médico cirujano y cirujano dentista antes de postular a los programas de formación (Pitak-Arnnop et al.).

Existe una cuarta área donde aún conviven en un mismo país 2 o más vías de formación para COMS, es el caso de Finlandia, Suecia, Australia y Nueva Zelanda, aunque todos ellos han decidido tomar la vía de la doble graduación como la oficial (Curtin, 2008; UEMS, 2008; Brennan et al., 2004, Szuster et al., 2000).

Actualmente se están produciendo muchos cambios en el marco del proceso de Bolonia tendiente a tener a un espacio europeo común de educación, esto 
ha llevado a que bajo la comisión de Cirugía OroMáxilo-Facial y Estomatología de la UEMS la especialidad se trabaje bajo directivas médicas, pero buscando lograr la doble-graduación como la única vía de formación de COMS y bajo el ideal de desarrollar la "armonización" (UEMS, 2007) en la formación de COMS en toda Europa, esto no solo con el fin de igualar programas, si no también en un intento de reducir el tiempo total de formación de un COMS, que en el caso de Inglaterra llega a 18 años una vez terminada la educación secundaria.

Requisitos de la International Association of Oral and Maxillofacial Surgeons. A pesar de las diferencias en la formación de COMS existen requisitos de base que son propuestos por la Asociación Internacional de Cirujanos Orales y Máxilofaciales para todos los programas de graduación (International Association of Oral and Maxillofacial Surgeons, 2001), estos son:

El punto de ingreso puede ser desde un grado médico o dental.

La duración y requerimientos específicos de los programas deben ser definidos por las Asociaciones nacionales o regionales mientras estén orientados a formar COMS que practiquen por completo el campo de acción de la especialidad y que se describe más adelante.

La máxima duración desde la obtención del primer grado (médico o dental) hasta el término de la especialidad debe ser de 8 años. Esto busca asegurar que los COMS desarrollen una carrera larga y productiva.

Los programas deben ser impartidos por COMS que muestren un compromiso con la educación y que aseguren las condiciones curriculares, de infraestructura y logísticas para impartir un programa de calidad.

Es responsabilidad del director (cargo individual) de cada programa que los especialistas egresados cumplan a cabalidad los estándares de calidad de la especialidad.

Se debe contar con una cantidad suficiente de casuística e infraestructura adecuada para la atención hospitalaria y ambulatoria.

El becado debe tener la suficiente preparación en medicina para evaluar al paciente en toda su economía, considerando el riesgo quirúrgico y anestési$\mathrm{co}$, con un adecuado entendimiento de:
Enfermedad sistémica: Conocimiento integral de varios sistemas orgánicos con un particular énfasis en la fisiología y patología cardiovascular, pulmonar, hepática, renal y endocrina. Así como estar familiarizado en el manejo de apoyos diagnósticos como electrocardiografía, imagenología máxilofacial y general y el diagnóstico de laboratorio clínico.

Evaluación del paciente: conocimiento y manejo para la realización de la historia clínica y el examen físico segmentario.

Terapéutica: incluyendo conocimiento de los agentes terapéuticos aceptados y empleados en el manejo médico y la acción de los fármacos utilizados en la atención de los pacientes quirúrgicos de la especialidad.

La experiencia central en cirugía (general) no puede ser menor a 1 año (12 meses).

Es fundamental para la adecuada formación de los COMS desarrollar una amplia experiencia en las áreas de cuidado crítico del paciente quirúrgico seriamente enfermo y el manejo de equipo del paciente severamente dañado.

El becado debe tener formación en odontología, que lo diferenciará de las otras especialidades quirúrgicas.

El becado debe ser expuesto a la totalidad del campo de acción de la especialidad, en un tiempo mínimo de 30 meses con al menos 12 meses en un cargo de responsabilidad quirúrgica como residente jefe. Y obtener una amplia experiencia como primer cirujano en todas las áreas de la especialidad.

Los programas de especialidad deben ofrecer de manera completa y progresiva una secuencia de atención de pacientes ambulatorios, hospitalarios y de emergencias.

El campo de acción de los COMS incluye, pero no se limita, a:

Patología y medicina oral, incluyendo el manejo de las enfermedades de la región oral y máxilofacial. ansiedad.

Cirugía dentoalveolar y manejo del dolor y la

Manejo del trauma máxilofacial, de tejidos blandos y duros. 
Manejo de los tumores benignos y malignos de la región.

Cirugía reconstructiva regional, incluyendo el transplante de tejidos blandos y duros, así como microcirugía.

Cirugía ortognática y ortopédica.

Cirugía facial estética.

Tratamiento quirúrgico de las anormalidades congénitas, incluyendo labio y paladar fisurado.

El becado debe recibir a lo largo del programa una amplia experiencia en el manejo anestésico regional y general, así como otros métodos de control del dolor y la ansiedad.

Los fellowships (subespecialidades) son el mejor método para proveer experiencia adicional en la ampliación del campo de acción de la especialidad.

El becado debe tener la oportunidad de discutir los planes de tratamiento con otros especialistas, tanto médicos como cirujano dentistas.

Regularmente deben agendarse seminarios y conferencias para aumentar el programa clínico. Así como la participación en congresos científicos nacionales e internacionales.

Con respecto a la evaluación del becado, esta debe ser permanente y en función de ella debe ser promovido a experiencias de mayor responsabilidad.

Los becados deben ser estimulados a participar en la investigación y publicación científica, siendo altamente recomendable que desarrollen un proyecto de investigación, así como brindar la posibilidad de obtener un grado académico (magíster o doctor).

Se debe promover el intercambio educativo de los becados en el plano internacional.

Rutas de formación y su duración. De las 3 vías de formación de COMS, la que presenta la ruta más simple es la single-degree odontológica, en ella el acceso al curso de la especialidad es de manera directa posterior a la titulación como cirujano dentista y en la mayor parte del mundo su duración es de 3 a 4 años (Goss et al., 1996).
La segunda vía, single-degree médica, presenta algunas variaciones: las que van desde un acceso inmediato a la especialidad, otras en que se debe cursar la especialidad de estomatología antes de la (sub)especialidad quirúrgica y por último otras en que a la anterior se puede agregar una formación previa de enfoque quirúrgico general. Todas estas variaciones llevan a que esta vía de formación tome de 3 a 6 años para convertirse en COMS luego de obtener el título médico (UEMS, 2007).

La tercera vía, dual-degree, es sin duda la que alcanza el mayor nivel de complejidad. Aquí encontramos vías de formación relativamente directas como la estadounidense, en que posterior al título odontológico se puede cursar un programa de 6 años que otorga el título (grado) médico y el de COMS (Dodson et al.), importante resaltar el ejemplo de Harvard que también permite el ingreso con un grado médico para en 6 años obtener el odontológico y COMS; otras vías separan claramente la obtención de los títulos médicos y odontológicos previo a cursar la especialidad, aunque aquí también vemos que algunos países tienen un tronco común, oficial (UEMS, 2007) o de facto (Maclver \& Chiu, 2005), para medicina y odontología con lo que el segundo título se obtiene en un tiempo menor, que generalmente es medicina y donde se puede cursar solo los últimos 3 años; finalmente esta vía alcanza un trayecto complejo en el caso de Inglaterra, donde posterior a la obtención del primer título se deben obtener una serie de grados (médico o dental ) y certificaciones que posteriormente permitirán al doble-graduado postular a la formación como COMS (Cameron \& Westcott, 2008; Langdon), el que luego deberá obtener una nueva certificación para poder ejercer a cabalidad la especialidad.

Con todo lo anterior, la vía de la doble-graduación toma de 6 a 13 años de formación adicional a la adquisición del primer grado, sea este médico u odontológico, lo que resalta la necesidad de la "armonización" para formar COMS doble graduado en un menor tiempo, lo que asegure una larga y productiva carrera clínica, académica o en la investigación y que redunde en un mayor desarrollo de la especialidad.

Competencias y prerrogativas clínicas. De todas las competencias que plantea la IAOMS, como parte integral del campo de acción de los COMS, la cirugía reconstructiva, oncológica y microcirugía son las que en prácticamente en todo el mundo, a excepción de Japón (Laskin), están reservadas solo a aquellos cirujanos que cuenten con un grado médico (Goss et 
al.) y preferentemente a aquellos con algún grado de subespecialización (fellowships) en cualquiera de dichas áreas, a las que igualmente se accede solo con el grado médico. Lo mismo sucede con otro tipo de procedimientos no-dentoalveolares, los que son en mayor cantidad realizados por COMS doble graduados (Brennan et al.). Recientemente se ha sugerido que el crecimiento de la especialidad, en un grado importante, es hacia la cirugía oncológica y que son los programas con directores o docentes con la subespecialidad quienes facilitan este desarrollo (Clark et al., 2011).

En este sentido las prerrogativas asociadas a dichas competencias siguen el mismo comportamiento, con lo que en una intervención quirúrgica que haga uso de técnicas microquirúrgicas y/o resectivas oncológicas y/o reconstructivas el cirujano líder casi siempre será un médico (Goss et al.; Herford et al., 2001), por lo que en muchos casos esa responsabilidad no recae en el cirujano máxilofacial (dentista), si no en el plástico, general o de cabeza y cuello (médico cirujano).

Lo anterior sin duda limita el campo de acción de la especialidad e impide un adecuado desarrollo de esta en todas sus posibilidades para ofrecer el tratamiento necesario a los pacientes con patologías máxilofaciales de forma más autónoma, lo que nuevamente resalta la importancia de formar COMS con la doble graduación.

Factores socioeconómicos. Si bien un amplio número de especialistas coinciden con las ventajas que tiene la formación de COMS doble graduados, también existe consenso que la duración y costos de esta formación son inalcanzables de abordar en muchos países, lo que también se da en el caso de muchos profesionales en países de mayores ingresos (UEMS, 2007).

Esto se ha comprobado en Estados Unidos, donde a pesar que la mitad de los programas y cupos son dual-degree, la mayoría de los postulantes buscan acceder a los programas single-degree, esto debido principalmente a los altos costos que tiene la formación adicional para la obtención del grado médico (Phan \& Davis, 2011).

Lo anterior no significa que los postulantes basen su decisión principalmente en el factor económico, si no que principalmente consideran la reputación de los programas, la existencia de abundante material clínico y una buena atmósfera de trabajo tanto con los docentes como con los becados de cursos superiores (Marciani et al., 2003). Es destacable mencionar que a pesar de las consideraciones económicas en la elección de un programa single o dualdegree, son quienes eligen este último los que presentan el mayor grado de satisfacción (Laskin et al., 2003).

Además debemos considerar que el mayor tiempo que demanda la obtención del grado médico tiene consecuencias a nivel familiar, en especial dado el rango etáreo de los postulantes. Otro hecho que refuerza la elección de los programas single-degree es que los COMS con consultas privadas ya establecidas prefieren cirujanos recién egresados con quienes puedan desarrollar relaciones interpersonales de buena calidad, que posean manejo en cirugía dentoalveolar, implantes y anestesia, por lo que en este caso no se prioriza el poseer el grado médico (Hinic et al., 2011).

Productividad científica. Se ha comprobado que los programas de doble graduación alcanzan una mayor productividad científica (Assael, 2010), a pesar de que existe evidencia (en Estados Unidos) que son los programas de single-degree los que ofrecen mayores posibilidades de participar en proyectos de investigación y publicación (Mohammad et al., 2011), lo que no se condice con los datos referentes a los programas que más publican (Assael, 2010).

A pesar de esta superioridad de los dualdegree no se tiene claridad si esto se debe a factores cualitativos (presencia de formación médica) o cuantitativos (mayor duración de los programas). Uno de los posibles factores es la relación entre la producción científica y el rendimiento académico en el pregrado, toda vez que los directores de los programas de doble grado privilegian más este último (Spina et al., 2000), por lo que sus becados promedian más alto en sus calificaciones y rankings de egreso del pregrado (Laskin et al.).

En el último tiempo la formación de COMS con intenciones de hacer carrera académica y competencias en investigación, e incluso la necesidad de grado académico, también se ha sumado al debate, toda vez que varios expertos han hecho notar un rezago en la investigación en cirugía oral y máxilofacial frente a otras especialidades dentales, médicas y quirúrgicas (Hupp, 2003; McKechnie \& McCaul, 2007). En este aspecto se ha identificado como factores 
predisponentes a la carrera académica contar con programas con un buen número de profesores y en especial que estos participen de proyectos de investigación (Sarraf et al., 2011).

Tendencia. En los últimos 20 años la formación mundial de COMS ha mostrado una clara tendencia a la progresiva doble-graduación, sin embargo, esto no se ha traducido en una exigencia como requisito imprescindible para ejercer, con carácter legal, en todos los países donde han aparecido este tipo de programas, razón por la que hoy en gran parte del mundo vemos una convivencia de COMS single (dentistas o médicos) y dual-degree. Esta convivencia ha generado una discusión importante en la comunidad internacional de COMS que, sin embargo, como hemos visto en esta revisión no ha definido una superioridad absoluta de una u otra vía de formación, principalmente porque el mejoramiento que se logra con la doble graduación lleva aparejado un aumento de los costos financieros que no siempre es posible abordar.

A todo este debate debemos sumar una serie de editoriales que contraponen las formaciones de base odontológica y con doble graduación, en ellas principalmente se ofrecen una serie de datos subjetivos, aunque no por ello ausentes de valor para la discusión.

En esta difusa definición de las vías de formación, la elección de mayor peso es la que ha hecho la Comunidad Europea a través de la UEMS y donde se ha albergado a la especialidad bajo directivas médicas, pero manteniendo la necesidad de contar con la formación odontológica, tal como lo plantea la IAOMS en sus requisitos.

En consideración de estas tendencias parece justo entender la doble-graduación como una evolución de la formación de COMS, en línea con una corriente internacional de cuestionamiento de la profesión odontológica, dado el perfil epidemiológico actual, la que aboga por una mayor presencia de la educación médica en la formación de los futuros dentistas (Tanaka et al., 2008; Rivera Peralta, 2001).

Realidad chilena. Actualmente en Chile, al igual que el resto de América Latina, no existe la posibilidad de formarse como COMS con doble graduación en un programa integrado, aunque claro está, existe la opción de cursar medicina y odontología en forma completa, con lo que un COMS chileno doble graduado demoraría 16 años en alcanzar la especialidad, lo que a la luz dela realidad internacional no parece excesivo, pero si altamente ineficiente ya que el contacto directo y profundo con la especialidad sería de apenas 3 años para los interesados en tomar ese camino, el que seguiría teniendo ausente una formación integral que permita practicar todo el campo de acción de la especialidad.

En estos momentos son 5 las universidades que dictan programas de formación de COMS, estas son: de Chile, de Valparaíso, de Concepción, Mayor, de Antofagasta y San Sebastian. Si analizamos sus programas de estudio vemos que ninguna de ellas cumple a cabalidad con los requisitos planteados por la IAOMS, en especial los referidos a plazos mínimos y la formación en cirugía oncológica, reconstructiva y microcirugía, estas últimas por la falta de formación médica.

Por otro lado, en años recientes han aparecido programas de formación de Cirujanos Orales en nuestro país, con un campo de acción limitado a la cirugía y traumatología dentoalveolar. Lo anterior desde un punto de vista de salud pública es una medida altamente eficiente, pues en un tiempo y con costos menores logra formar un especialista que da respuesta a las patologías más prevalentes que ahora tratan los COMS. Sin embargo, todo esto, por una parte pone en duda el real espacio que tendrán los COMS y por otro refuerza la necesidad de que en el país se logre desarrollar la especialidad en todo su campo de acción reconocido internacionalmente, lo que a la luz de la evidencia expuesta pasa necesariamente por contar con programas de doble grado.

Observaciones finales. En el escenario internacional la doble graduación en cirugía oral y máxilofacial no aparece como simplemente otra opción más en la formación de estos especialistas, si no como una tendencia potente y cierta para el aseguramiento de la mejor atención posible de los pacientes y el desarrollo de la especialidad y sus cultores. Lo anterior en ningún caso es subvalorar el trabajo que han tenido los COMS con solo el grado dental, es un llamado a promover la evolución de la especialidad.

Si bien en la actualidad parece improbable que en nuestro país a corto plazo se desarrollen programas de doble graduación, es deber de todos los actores involucrados considerar y discutir acerca de esta tendencia evolutiva, de manera que la cirugía oral y máxilofacial chilena se mantenga en los estándares internacionales. 
CARTES-VELÁSQUEZ, R. \& RAMÍREZ, S. H. Dual-degree oral and maxillofacial surgery. Int. J. Odontostomat., 5(2):191198, 2011.

\begin{abstract}
Oral and maxillofacial disease profile is treated by overlapping medical and surgical specialties, most of them without the high-level stomatological training of a surgeon dentist. Of these specialties, oral and maxillofacial surgery (OMS) is the only one that requires medical, dental and surgical training, which currently is achieved by 3 main routes. To date, the debate continues about which of them is the ideal, although in Latin America the only way to become an OMS specialist is the odontological. A critical review of international literature in OMS journals and guidelines of international societies, focusing on the bioethical topics, clinical competences, educational, legal and social aspects were made. Dualdegree is advantageous to the WHO practice because of the following aspects: clinical scope (oncological and reconstructive surgery), access to fellowships and scientific-professionals societies, knowledge/evidence generation, surgical prerogatives, legal support and social acknowledgement. From a bioethical perspective, single-degree specialists dealing with maxillofacial pathology may exceed their competences, due to a lack of adequate medical or dental training. The dual-degree specialist seems to be the one, from a comprehensive perspective of the patient, has the knowledge and skills to fully meet the community's rights to healthcare and contribute to specialized development. Unfortunately, Latin America has no dualdegree training programs and none of Chilean single-degrees fullfil the IAOMS guidelines. This review exposes the need to develop a dual-degree program in Chile to achieve a comprehensive approach in the patient with maxillofacial pathology; consistent with the social, cultural, economic and educational reality of the country.
\end{abstract}

KEY WORDS: surgery, maxillofacial, dual-degree, review, Chile.

\title{
REFERENCIAS BIBLIOGRÁFICAS
}

Assael, L. A. Training the future: Protecting the scope and diversity of oral and maxillofacial surgery. J. Oral Maxillofac. Surg., 65(2):161-2, 2007.

Assael, L. A. The view from the third rail. J. Oral Maxillofac. Surg., 68(4):713-4, 2010.

Brennan, D. S.; Spencer, A. J.; Singh, K. A.; Teusner, D. N. \& Goss, A. N. Practice patterns of oral and maxillofacial surgeons in Australia: 1990 and 2000. Int. J. Oral Maxillofac. Surg., 33(6):598-605, 2004.

Cameron, M. \& Westcott, L. Maxillofacial training is no longer than other surgical specialties. Ann. R. Coll. Surg. Engl., 90(2):146-9, 2008.

Clark, P.; Markiewicz, M.; Bell, B. \& Dierks, E. Trends and Attitudes Regarding Head and Neck Oncologic Surgery: A Survey of United States Oral and Maxillofacial Surgery Programs. J. Oral Maxillofac. Surg., doi:10.1016/j.joms.2011.02.113, 2011.

Cruz-Coke, M. R. Peregrinaciones a las fuentes de la medicina clásica. Rev. Med. Chile, 135(8):1076-81, 2007.

Curtin, J. OMS training in Australia and New Zealand. J. Oral Maxillofac. Surg., 66(10):2193, 2008.

Dodson, T. B.; Guralnick, W. C.; Donoff, R. B. \& Kaban, L. B. Massachusetts General Hospital/Harvard Medical School MD oral and maxillofacial surgery program: a 30-year review. J. Oral Maxillofac. Surg., 62(1):62-5, 2004.

Goss, A. N.; Helfrick, J. F.; Szuster, F. S. \& Spencer, A. J. The training and surgical scope of oral and maxillofacial surgeons: the International Survey 1994. Int. J. Oral Maxillofac. Surg., 25(1):74-80, 1996.

Goudot, P. \& Bonnel, M. Livre blanc de la stomatologie et chirurgie maxillo-faciale Française. France, ESV production, 2006.

Herford, A. S.; Pulsipher, D. A. \& Sinn, D. P. Integration of the Medical Degree in Oral and Maxillofacial Surgery: A 10-Year Follow-Up. J. Oral Maxillofac. Surg., 59(12):1471-6, 2001.

Hinic, P.; Johnson, M. P. \& Kelly, J. P. Desired Characteristics of a Potential Oral and Maxillofacial Surgery Practice Associate. A Connecticut Survey Response. J. Oral Maxillofac. Surg., doi:10.1016/ j.joms.2011.02.055, 2011.

Hupp, J. R. An Academic Career in Oral and Maxillofacial Surgery: Societal Impact and Financial Considerations. J. Oral Maxillofac. Surg., 61(7):82933, 2003.

International Association of Oral and Maxillofacial Surgeons. International Training Guidelines. Last updated in 2001. Disponibles en http://www.iaoms.org/ Training Guidelines. aspx?display=Training 
Langdon, J. Training for Oral and Maxillofacial Surgery, Academic Oral Surgery, and Surgical Dentistry in the United Kingdom. J. Oral Maxillofac. Surg., 64(12):1803-6, 2006.

Laskin, D. M.; Lesny, R. J. \& Best, A. M. The Residents Viewpoint of the Matching Process, Factors Influencing Their Program Selection, and Satisfaction with the Result. J. Oral Maxillofac. Surg., 61(2):228-33, 2003.

Laskin, D. The Past, Present, and Future of Oral and Maxillofacial Surgery. J. Oral Maxillofac. Surg., 66(5):1037-40, 2008.

Legrá Matos, S. M.; Milanés Pérez, O.; Comas Mirabent, R.; López Bancourt, A. C. \& Carreras Martorell, C. L. Antecedentes y perspectivas de la Estomatología en Cuba. MEDISAN, 10, 2006. Disponible en: http:// bvs.sld.cu/revistas/san/vol10_e_06/san05(esp)06.pdf

Maclver, C. \& Chiu, G. A. Preparing for a career in oral and maxillofacial surgery: a survey of dentists at medical schools. Br. J. Oral Maxillofac. Surg., 43(6):516-9, 2005.

Mahmood, S.; MacLeod, S. P. \& Lello, G. E. FRCSEd (Oral and Maxillofacial Surgery): a milestone in the history of Oral and Maxillofacial Surgery in the United Kingdom. Br. J. Oral Maxillofac. Surg., 40(4):300-3, 2002.

Marciani, R. D.; Smith, T. A. \& Heaton, L. J. Applicants' Opinions About the Selection Process for Oral and Maxillofacial Surgery Programs. J. Oral Maxillofac. Surg., 61(5):608-14, 2003.

McKechnie, A. \& McCaul, J. Research training for oral and maxillofacial surgery. Br. J. Oral Maxillofac. Surg., 45(6):478-83, 2007.

Mohammad, A. E.; Best, A. M. \& Laskin, D. M. Attitudes and Opinions of Residency Directors and Residents About the Importance of Research in Oral and Maxillofacial Surgery Residencies. J. Oral Maxillofac. Surg., 69(7):2064-9, 2011.

Moreno-Altamirano, L. Reflexiones sobre el trayecto salud-padecimiento-enfermedad-atención: una mirada socioantropológica. Salud Pública Méx., 49(1):63-70, 2007.

Phan, T. T. \& Davis, J. M. Demand for single- and dualdegree oral and maxillofacial surgery residency positions. J. Oral Maxillofac. Surg., 69(1):242-7, 2011.
Pitak-Arnnop, P.; Bauer, U.; Chaine, A.; Hemprich, A. \& Bertolus, C. The past, present, and future of oral and maxillofacial surgery--some details in Europe. J. Oral Maxillofac. Surg., 68(2):491-4, 2010.

Rivera Peralta, H. Estomatología, La Odontología del III Milenio Reflexiones de 50 años de profesión 19512001. Santiago, Autoedición, 2001.

Sarraf, A. A.; Abubaker, A. O.; Laskin, D. M. \& Best, A. M. Characteristics of Oral and Maxillofacial Surgery Residencies That Result in Graduating Residents Entering Academic Positions. J. Oral Maxillofac. Surg., 69(8):2271-4, 2011.

Schapira, M. V. La odontología en Argentina: historia de una profesión subordinada 'La práctica odontológica: de la consolidación profesional a las transformaciones de los años 1960'. Hist. Cienc. SaudeManguinhos, 10(3):955-77, 2003.

Scott, J. Dental education in Europe: the challenges of variety. J. Dent. Educ., 67(1):69-78, 2003.

Spina, A. M.; Smith, T. A.; Marciani, R. \& Marshall, E. O. A survey of resident selection procedures in oral and maxillofacial surgery. J. Oral Maxillofac. Surg., 58(6):660-6, 2000.

Szuster, F. S.; Nastri, A. L.; Goss, A. N. \& Spencer, A. J. Survey of Australian and New Zealand Oral and Maxillofacial Surgery trainees and recent specialists-education and experience. Int. J. Oral Maxillofac. Surg., 29(4):305-8, 2000.

Tanaka, K.; Honda, T. \& Kitamura, K. Dentistry in Japan should become a specialty of medicine with dentists educated as oral physicians. J. Dent. Educ., 72(9):1077-83, 2008.

UEMS. Section of Oro-Maxillo-Facial Surgery. Minuta 2007. Bruselas, Bélgica, The European Union of Medical Specialties, 2007.

UEMS. Section of Oro-Maxillo-Facial Surgery. Minuta 2008. Bruselas, Bélgica, The European Union of Medical Specialties, 2008.

Dirección para correspondencia:

Ricardo Cartes-Velásquez

Los Olivos 37, Penco.

CHILE

Email: ricardo@cartesvelasquez.com

Recibido : 27-07-2011 Aceptado: 12-08-2011 